\title{
Outcomes After Liver Resection for Hepatic Alveolar Echinococcosis: A Single-Center Cohort Study
}

\author{
Gaëtan-Romain Joliat ${ }^{1} \cdot$ Emmanuel Melloul $^{1} \cdot$ David Petermann $^{1} \cdot$ \\ Nicolas Demartines ${ }^{1} \cdot$ Michel Gillet $^{1} \cdot$ Emilie Uldry $^{1} \cdot$ Nermin Halkic $^{1}$
}

Published online: 12 June 2015

(C) Société Internationale de Chirurgie 2015

\begin{abstract}
Background Switzerland is a region in which alveolar echinococcosis (AE) is endemic. Studies evaluating outcomes after liver resection (LR) for AE are scarce. The aim of this study was to assess the short- and long-term outcomes of AE patients after LR in a single tertiary referral center.

Methods We retrospectively analyzed data pertaining to all patients with liver AE who were treated with LR at our institution between January 1992 and December 2013. Patient demographics, intraoperative data, extent of LR procedures (major vs. minor LR), postoperative outcomes, and negative histological margin (R0) resection rate were recorded in a database. Recurrence rates after LR were analyzed.

Results LR was performed in 59 patients diagnosed with hepatic AE (56 complete surgeries, 3 reduction surgeries). Postoperative morbidity and mortality were observed in $34 \%$ ( $25 \%$ grade I-II, $9 \%$ grade III-IV) and $2 \%$ of the patients, respectively. R0 (complete) resection rate was $71 \%(n=42)$, and R1/R2 resection rate was $29 \%(n=17)$. Extra-hepatic recurrence occurred in 1 case (lung) after R0 resection. In cases of R1/R2 resection, 7 intra-hepatic disease progressions occurred with a median time of 10 months (IQR 6-11 months). Long-term (more than 1 year) benzimidazole treatment stabilized the disease in $64 \%$ (9/14) of patients with R1 status. The overall survival rate was $97 \%$.

Conclusions Liver AE can be safely and definitively treated with LR, provided that R0 resection is achieved. In cases of R1 resection, benzimidazole therapy seems to be effective in stabilizing the intra-hepatic disease and preventing extra-hepatic recurrence.
\end{abstract}

\section{Introduction}

Alveolar echinococcosis (AE) is a rare parasitosis due to the larval form of Echinococcus multilocularis [1-3]. In Europe, Switzerland is considered to be an endemic region for this parasite, as are France, Germany, or Austria [4].

Gaëtan-Romain Joliat gaetan.joliat@gmail.com

$\triangle$ Nicolas Demartines demartines@chuv.ch

1 Department of Visceral Surgery, University Hospital CHUV, Rue du Bugnon 46, 1011 Lausanne, Switzerland
AE can be compared to a malignant tumor because of its invasiveness and ability to infiltrate not only the liver parenchyma, but also other organs such as lungs or brain. Similar to the TNM classification system used to describe the stages of cancer progression, a PNM classification for $\mathrm{AE}$ was developed by the World Health Organization (WHO). This classification is based on the location of the liver lesion, invasion of the vascular/biliary structures $(\mathrm{P})$, the involvement of neighboring organs $(\mathrm{N})$, and the presence of distant metastasis (M) [5]. Since AE mainly affects the liver, symptoms at the time of diagnosis are similar to liver cancer. Patients may have abdominal pain, jaundice, and/or weight loss [6]. If left untreated, approximately $95 \%$ of $\mathrm{AE}$ patients will succumb [7]. 

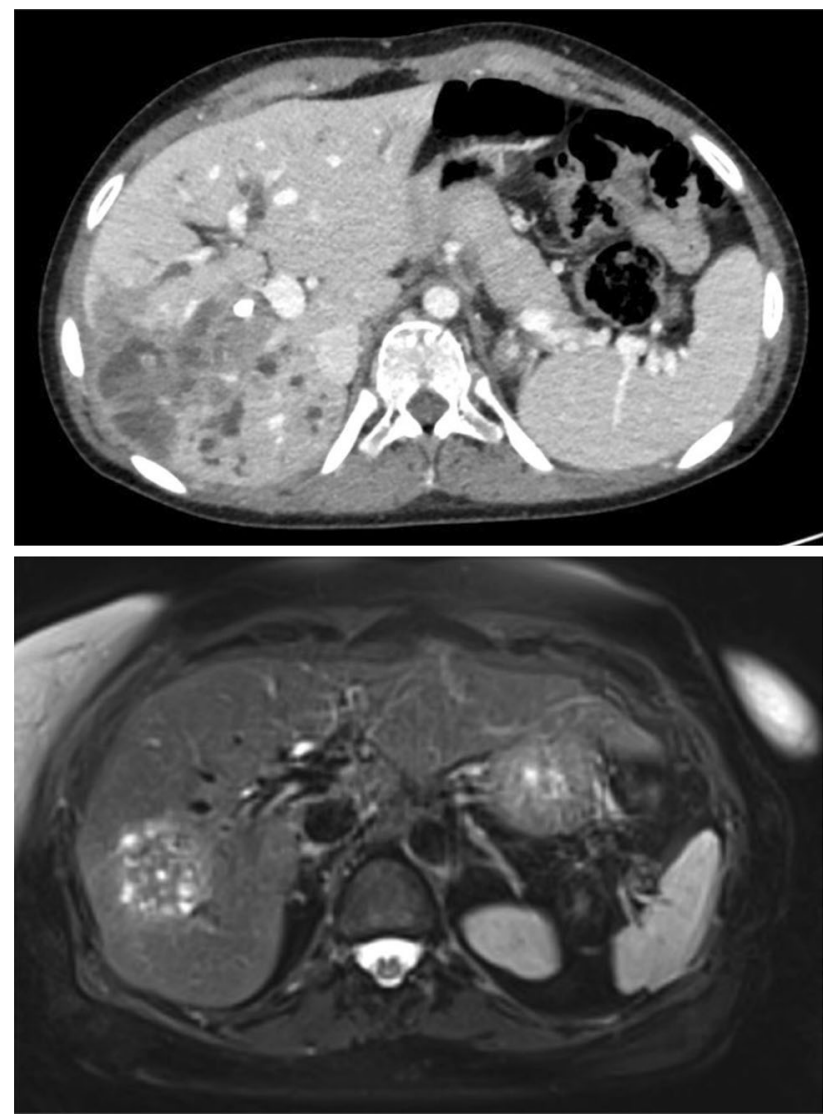

Fig. 1 Typical CT-scan and MRI axial views of hepatic alveolar echinococcosis

Therefore, aggressive therapy including major liver resection (LR) is often necessary to eradicate the totality of the parasites.

To date, few studies have been published on postoperative outcomes after LR for AE [6, 8-13]. The largest surgical series previously published was a study conducted in Japan, which included a large number of patients with incomplete resection of the hepatic disease [6]. Western series are scarce and these studies include a mixture of debulking strategies, medical treatments, and complete resections [9, 12]. The aim of this study was to evaluate the short- and long-term results of LR for AE in a tertiary referral center located in an area in which $\mathrm{AE}$ is endemic. The recurrence rate was analyzed and compared between cases in which negative (R0), positive histological (R1), and positive macroscopic (R2) resection margins were achieved.

\section{Materials and methods}

We conducted a retrospective analysis of all consecutive patients surgically treated for liver AE at the University Hospital of Lausanne (CHUV) from 1992 to 2013 based on prospective patient data. Preoperative patient and lesion characteristics, preoperative treatments, intraoperative data, and postoperative results were analyzed. The study was granted prior approval by the local ethics committee (protocol number 56/14).

\section{Preoperative work-up}

Prior to surgery, all patients had a high-resolution contrastenhanced CT-scan of the chest and abdomen to assess liver volume and vascular anatomy, to measure the extent of the hepatic lesion, and to rule out extra-hepatic disease. In cases where remnant liver volume was going to be less than $30 \%$ of the total volume of the normal liver parenchyma, preoperative portal vein embolization (PVE) was performed according to the standard technique. Patients with preoperative biliary obstruction and jaundice underwent percutaneous or endoscopic drainage prior to surgery. The primary lesions were classified according to the PNM classification system for AE developed by the WHO in 2006 [5]. In this system, the "P stage" defines the liver location of the lesion and indicates whether the vascular/biliary structures have been invaded. The "N stage" concerns the involvement of the neighboring organs, and "M stage" indicates whether any distant metastasis is present [5]. If a suspicious image was found on CT-scan (Fig. 1) and the patient was either living in an endemic area or had other risk factors, then screening for AE was performed. Screening and work-up included serology testing (Western blot, ELISA) and liver MRI (Fig. 1) [14, 15].

\section{Surgical procedure}

Major LR was defined as the resection of three or more Couinaud's segments. The surgical procedure consisted of parenchymal transection using an ultrasonic dissector. Intermittent pedicle clamping without preconditioning (Pringle maneuver) was reserved only for cases in which there was bleeding. Hemostasis was achieved with bipolar coagulation, hemoclips, and ligatures. Debulking R2 resection was performed in symptomatic patients where the macroscopic disease could be maximally removed, as determined by preoperative work-up and multidisciplinary discussion.

\section{Postoperative data and patient follow-up}

Postoperative complications were graded according to the Dindo-Clavien classification [16]. As AE behaves like a cancer, the resection margins were defined the same as for a LR for hepatocellular carcinoma. R0 resections were defined as those in which the absence of disease was confirmed in the resection margins, and $\mathrm{R} 1$ resections were 
Table 1 Patient characteristics

\begin{tabular}{ll}
\hline & $\begin{array}{l}\text { Patients } \\
n=59\end{array}$ \\
\hline Age $^{\mathrm{a}}$ & $60(49-70)$ \\
Women & 29 \\
Symptoms at diagnosis & 39 \\
Metastasis at diagnosis & 8 \\
Intent of curative resection & 56 \\
Debulking R2 resection $^{\mathrm{b}}$ & 3 \\
Preoperative treatment $^{\mathrm{b}}$ & 20 \\
\hline
\end{tabular}

a Median with interquartile range

b Radiological, endoscopic, or medical (benzimidazole treatment $>6$ months before the operation)

Table 2 Preoperative lesion characteristics $(n=59)$

\begin{tabular}{ll}
\hline PNM stage I/II/III/IV & $26 / 3 / 16 / 14$ \\
Largest liver lesion size $^{\mathrm{a}}(\mathrm{cm})$ & $5.5(3.9-9.8)$ \\
Vena cava invasion & 2 \\
Portal vein invasion & 8 \\
Hepatic vein invasion & 8 \\
Hepatic artery invasion & 8 \\
Diaphragm invasion & 13 \\
Biliary tree invasion & 16
\end{tabular}

a Median with interquartile range

defined as resections with positive histological margins $(<1 \mathrm{~mm})$. R2 resections were defined as palliative resections with macroscopic positive margins (debulking). Recurrence was defined as the appearance of intra-hepatic disease at or distant to the resection site after R0 resection, or new extra-hepatic disease. Progression of the disease was defined as intra-hepatic progression observed on radiological studies after a $\mathrm{R} 1$ or $\mathrm{R} 2$ resection was performed.

Patients were examined at the outpatient clinic 1 month after surgery. Subsequent follow-up was performed either by the patient's general practitioner or by a staff surgeon at our institution. Follow-up included clinical examination, serological tests, and imaging.

\section{Benzimidazole therapy}

Preoperatively, as soon as the diagnosis of $\mathrm{AE}$ was obtained, albendazole treatment was initiated if there was no contraindication. In cases where R0 resection was achieved, albendazole treatment was given for 2 years after the operation. Long-term (life-long) albendazole treatment was pursued when R1 or R2 resections occurred. In cases where albendazole intolerance or hepatic toxicity occurred, mebendazole treatment was given. No benzimidazole
Table 3 Surgical results

\begin{tabular}{lr}
\hline Minor resection & 24 \\
Major resection & 35 \\
Pedicular clamping & 9 \\
Vascular resection & 7 \\
Diaphragm resection & 13 \\
Biliary reconstruction (duct-to-duct or Roux-en-Y anastomosis) $^{\mathrm{a}}$ & 9 \\
\hline${ }^{a}{ }^{2}$ Seven cases of AE invading the biliary tree did not need a biliary \\
reconstruction
\end{tabular}

Table 4 Postoperative results

\begin{tabular}{|c|c|c|}
\hline $\mathrm{R} 0$ & 42 & $71 \%$ \\
\hline $\mathrm{R} 1$ & 14 & $24 \%$ \\
\hline $\mathrm{R} 2$ & 3 & $5 \%$ \\
\hline Complications (Dindo-Clavien) & 21 & $36 \%$ \\
\hline Minor (I-II) & 15 & $25 \%$ \\
\hline Major (IIIa-IVb) & 5 & $9 \%$ \\
\hline Mortality (V) & 1 & $2 \%$ \\
\hline Overall mortality rate ${ }^{\mathrm{a}}$ & 2 & $3 \%$ \\
\hline Recurrences/disease progressions & 8 & $14 \%$ \\
\hline Intra-hepatic & 7 & $12 \%$ \\
\hline Extra-hepatic & 1 & $2 \%$ \\
\hline After R0 & 1 & $2 \%$ \\
\hline After R1 & 5 & $9 \%$ \\
\hline After R2 & 2 & $3 \%$ \\
\hline Follow-up ${ }^{\mathrm{b}}$ (months) & 84 & $(41-148)$ \\
\hline Postoperative benzimidazole treatment & 33 & $56 \%$ \\
\hline No recurrence after $\mathrm{R} 1 / \mathrm{R} 2$ resection & 10 & $17 \%$ \\
\hline No recurrence after $\mathrm{R} 1$ resection & 9 & $15 \%$ \\
\hline No recurrence after $\mathrm{R} 2$ resection & 1 & $2 \%$ \\
\hline
\end{tabular}

${ }^{a}$ Including the 30 postoperative day mortality

b Median with interquartile range

treatment was given if patients experienced allergy, hypersensitivity, intolerance, or during the first trimester of pregnancy. Before 2003, benzimidazole treatment was considered only in case of $\mathrm{R} 1$ or $\mathrm{R} 2$ resection.

\section{Statistical analyses}

All statistical analyses were performed using GraphPad Prism 5 for Mac OS X®. Survival curve calculation was conducted using the Kaplan-Meier technique.

\section{Results}

Patient characteristics are summarized in Table 1. At the time of diagnosis, two-thirds of the patients had symptoms (mainly abdominal pain) and $14 \%$ (8/59) exhibited distant 


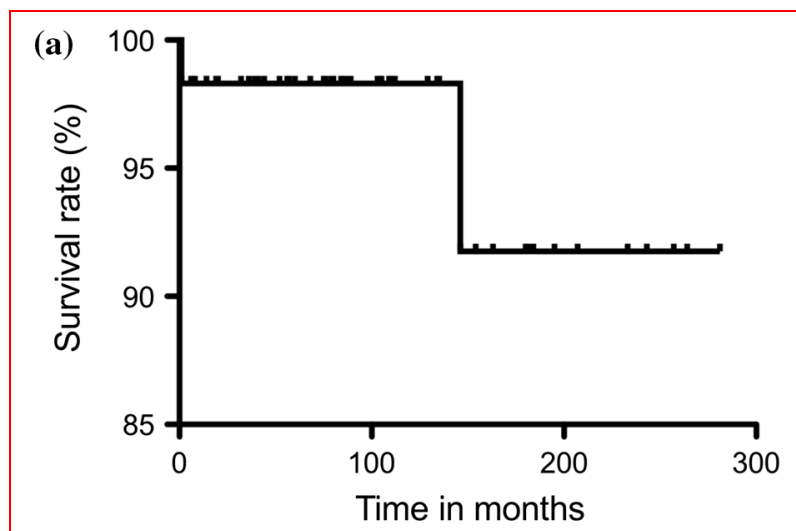

Alive patients

59

58

57

57

(b)

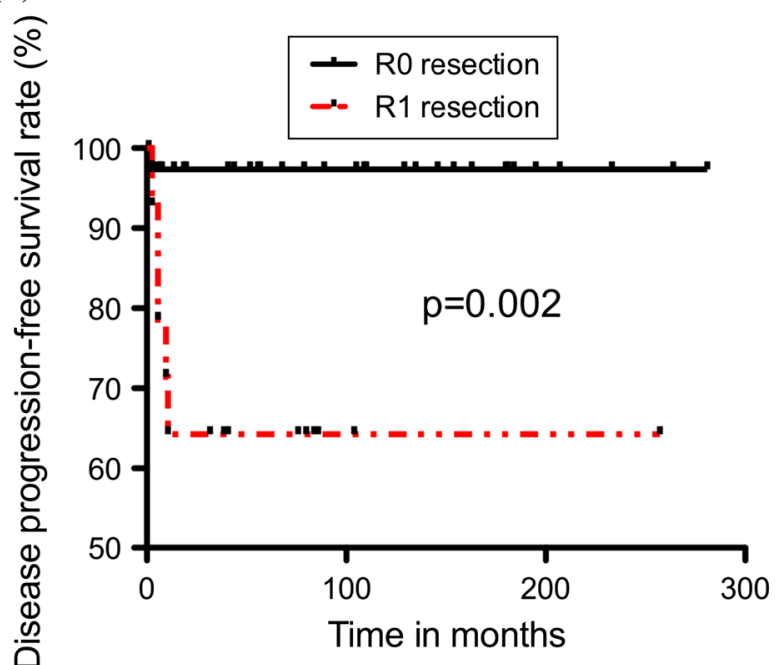

Patients without recurrence or disease progression
$\mathrm{RO}$

R1

\begin{abstract}
42
\end{abstract}
14
41

9
41

9
41

9
Fig. 2 Kaplan-Meier survival curve for alveolar echinococcosis surgical patients (a) and disease progression-free survival for patients with R0 and R1 resections (b)

metastasis. The main location of extra-hepatic disease was determined to be the lung ( 5 cases), followed by spleen and peritoneum. Surgery with curative intent was performed in 56 cases; only 3 patients had a debulking $\mathrm{R} 2$ resection. Biliary drainage was performed before surgery in 7 cases ( 5 by endoscopy, 2 percutaneously).

Serology for Echinococcus multilocularis was available for 40 patients $(68 \%)$. Before surgery, 35 patients had positive tests for Echinococcus multilocularis by Western blot (with 5 false-negative tests) and 32 had positive Em2antigen and EmII/3-10 antigen tests (with 8 false-negative tests). Of note, 12 patients also tested positive for Echinococcus granulosus by Western blot.
Table 2 shows the preoperative lesion characteristics. The most frequently observed PNM stage was stage I, followed by stage III, IV, and II, respectively. Forty-four percent of the patients (26/59) exhibited caval/portal/hepatic vein or hepatic artery invasion at the time of diagnosis. Major LR was performed in 35 cases (Table 3). A total of 16 patients presented with biliary invasion, but only 9 of them required bile duct resection with Roux-en-Y reconstruction or with duct-to-duct anastomosis (Table 3). Finally, additional diaphragm resection was necessary in 13 cases due to adjacent AE invasion (Table 3).

Table 4 summarizes the postoperative results. Major complications (grade IIIa-IVb) occurred in 5 cases, and 1 patient died during the postoperative period. The major complications seen included 2 postoperative hemorrhages, 1 case of septic shock due to colon perforation, 1 pulmonary embolism, and 1 biliary leak. The only postoperative death (grade V) was due to colon ischemia complicated by septic shock.

Histopathology showed that there were $71 \%$ R0 (42/ 59), $24 \%$ R1 (14/59), and $5 \%$ R2 (3/59) resections. During the follow-up (median: 84 months, IQR: 41-148 months), no patient had intra-hepatic recurrence after R0 resection, and only $2 \%$ (1/42) developed lung metastasis 4 months after surgery. After R1 and R2 resections, $41 \%(7 / 17, \mathrm{R} 1: 5 / 14, \mathrm{R} 2: 2 / 3)$ of the patients exhibited intra-hepatic disease progression (4 at the resection site, 3 in the biliary tree), and no patient exhibited new extra-hepatic lesion. The median time to disease progression for non-R0 resections was 10 months (IQR 6-11 months). For R1 resections, the median time to disease progression was 6 months (IQR 4.5-10.5 months) and for $\mathrm{R} 2$ resections it was 22.5 months (IQR 10-35 months). Three patients underwent a second LR and 1 underwent a Roux-en-Y anastomosis. In 20 cases, benzimidazole therapy was not conducted, but reasons why benzimidazole treatment was not given were missing. Among the remaining 39 patients, postoperative benzimidazole treatment (either 2-year or long-term therapy) was given in 33 cases. One patient declined the postoperative benzimidazole treatment and 5 had contraindications that resulted in discontinued therapy ( 2 cases of intolerance, 1 of allergy, 1 of hepatic toxicity, and 1 of first trimester of pregnancy). Albendazole was given in 31 cases and mebendazole in 2 cases. Among the R1 cases, benzimidazole treatment stabilized the disease in $9 / 14$ patients (64\%), meaning that 9 cases did not show disease progression after $\mathrm{R} 1$ resections and benzimidazole treatment $(15 \%$ of all patients, Table 4). After $\mathrm{R} 2$ resection, benzimidazole treatment stabilized the disease in $1 / 3$ patients (33\%).

As shown in Fig. 2, the overall survival rate was $97 \%$. There was 1 death due to a cerebral tumor not related to echinococcosis and 1 death due to septic shock during the 
first 30 postoperative days. Patients with R1 and R2 resections had $100 \%$ survival. The survival times observed in the 3 cases with debulking $\mathrm{R} 2$ resection were 103, 110, and 132 months.

\section{Discussion}

This study shows that LR allows a curative treatment for patients with resectable hepatic AE. The long-term results of $\mathrm{R} 1$ and debulking $\mathrm{R} 2$ resections are also acceptable provided that patients are treated with adjuvant benzimidazole therapy and closely monitored, as $100 \%$ of patients from this cohort survived after a median follow-up of 7 years

Due to its low worldwide incidence, data on long-term outcomes after LR for AE are scarce [6, 8-13, 17]. Hepatic $\mathrm{AE}$ exhibits a tumor-like behavior with liver invasion, destruction of the parenchyma, and infiltration of adjacent vascular or biliary structures [6]. Most of the symptoms, observed in two-thirds of patients, are related to the infiltrating behavior of this parasitosis [18]. These main symptoms comprise abdominal pain that is most often located in the right upper quadrant, jaundice, weight loss, and vomiting. Postoperative complications occurred in $36 \%$ of patients, mostly minor (i.e., grade I or II). The majority of the complications observed were respiratory, including pulmonary embolism, pneumonia, atelectasis, and pleural effusion. This incidence of common complications is related to the extensive nature of the surgery, as a high rate of major LR was performed in this cohort to obtain curative treatment of the parasitosis.

According to the PNM classification, the predominant stage of disease observed in this cohort was stage I. Since Switzerland is one of the major endemic regions of the disease, we adopted a low threshold policy with respect to diagnostic testing, performing $\mathrm{AE}$ serology, computed tomography, and/or magnetic resonance imaging in patients with a suspected liver cystic lesion [19]. Consequently, diagnosis was made early in the course of the disease, which allowed curative treatment in up to $71 \%$ of patients.

In this study, extra-hepatic recurrence in the lung appeared in only $2 \%$ of patients, and 7 patients showed disease progression after $\mathrm{R} 1$ or $\mathrm{R} 2$ resection, with a median time of recurrence of 10 months. These findings support previously published results suggesting that the remaining parasite needs several months to develop sufficiently to induce a recurrence $[6,12]$. Interestingly enough, in our cohort, no patient presented a brain metastatic lesion which is one of the most common locations of extra-hepatic alveolar echinococcosis [18].

The only curative treatment for hepatic $\mathrm{AE}$ remains surgery, and surgery is curative provided that a R0 resection is achieved $[1,6,9,10,12,13,17]$, in which case a long-term survival up to $97 \%$ is observed. In this study, the level of recurrence after $\mathrm{R} 0$ resection was only $2 \%$, whereas $41 \%$ of patients with $\mathrm{R} 1$ and $\mathrm{R} 2$ resections showed intra-hepatic disease progression. Alternatives to surgery include benzimidazole drug therapy (albendazole or mebendazole), endoscopic treatment (e.g., biliary drainage), or radiologic treatment (e.g., percutaneous biliary drainage) [20, 21]. In studies assessing benzimidazole treatment alone for hepatic $\mathrm{AE}$, the mortality rates range from 25 to $42 \%$ (with a median follow-up period of 12 years) [12, 20]. Endoscopic treatment of biliary complications related to $\mathrm{AE}$ seems to be less effective than for the treatment of hydatic cysts because of the malignant invasion of the parasite into the biliary tree [22, 23]. However, the endoscopic option can be used to treat cholangitis and jaundice prior to surgery. In the present study, $12 \%$ of patients were treated successfully by endoscopy or by percutaneous drainage before surgery. In addition, interventional radiology treatment may be indicated in case of hepatic vein involvement and Budd-Chiari syndrome [24]. Thus, treatment should be tailored for each patient on a case-by-case basis, using a multidisciplinary approach [6]. A WHO working group published, in 2010, treatment recommendations for $\mathrm{AE}$ in humans [25].

Hepatic R1 resections and debulking $\mathrm{R} 2$ resections in conjunction with postoperative benzimidazole therapy can also yield acceptable results $[6,26]$. The survival times of the 3 patients who underwent debulking R2 LR were 103, 110, and 132 months. However, these results have to be taken with caution, since 2 out of the 3 patients with debulking $\mathrm{R} 2$ resections showed a disease progression during the study period. In addition, all patients with R1 resection remained alive during the 7-year median followup period. Some disagreements exist in the published literature regarding whether or not palliative surgery is advisable. Some previous studies found that it is associated with worse long-term results $[12,13]$, whereas the current study agrees with other findings [6] that indicate that palliative surgery can improve long-term survival despite the fact that disease progression was $66 \%$ even with benzimidazole therapy.

Western blot tests for E. multilocularis gave false-negative results in $13 \%$ of the cases (5/40), while ELISA testing with Em2-antigens and EmII/3-10 antigens gave $20 \%$ false-negative results $(8 / 40)$. This observation corroborates the conclusions from other studies that the Western blot-based test provides more sensitive detection of E. multilocularis than ELISA [18, 27, 28]. Of note, due to cross-reactivity, positive Western blot tests for $E$. $m u l$ tilocularis can give positive results for E. granulosus (observed in 12 out of 40 patients) [29]. The use of Em2-and EmII/3-10 antigen ELISA tests coupled to Western blot- 
based diagnosis thus increases the sensitivity and specificity of E. multilocularis detections in cases of clinical and radiological suspicion [29]. In case of negative E. multilocularis-specific serology, some authors advocate the use of ${ }^{18}$ F-FDG PET-CT to monitor the efficacy of postoperative benzimidazole treatment and to assess the possibility of treatment discontinuation [30].

Surgery remains the treatment of choice of AE if the totality of the parasitic lesions can be removed. LR for hepatic $\mathrm{AE}$ is curative with an acceptable morbidity and mortality, provided that a $\mathrm{R} 0$ resection can be achieved. R1 resection coupled with benzimidazole adjuvant treatment is associated with acceptable long-term results by controlling microscopic hepatic disease. Debulking R2 resections still are matter of discussion but they can yield acceptable longterm survival even though the risk of disease progression is high.

Conflict of interest The authors have no conflicts of interest or financial ties to disclose.

\section{References}

1. Eckert J, Deplazes P (2004) Biological, epidemiological, and clinical aspects of echinococcosis, a zoonosis of increasing concern. Clin Microbiol Rev 17:107-135

2. Ammann RW, Eckert J (1996) Cestodes. Echinococcus. Gastroenterol Clin North Am 25:655-689

3. Craig P (2003) Echinococcus multilocularis. Curr Opin Infect Dis $16: 437-444$

4. Schweiger A, Ammann RW, Candinas D et al (2007) Human alveolar echinococcosis after fox population increase, Switzerland. Emerg Infect Dis 13:878-882

5. Kern P, Wen H, Sato N et al (2006) WHO classification of alveolar echinococcosis: principles and application. Parasitol Int 55(Suppl):S283-S287

6. Kawamura N, Kamiyama T, Sato N et al (2011) Long-term results of hepatectomy for patients with alveolar echinococcosis: a single-center experience. J Am Coll Surg 212:804-812

7. Torgerson PR, Schweiger A, Deplazes P et al (2008) Alveolar echinococcosis: from a deadly disease to a well-controlled infection. Relative survival and economic analysis in Switzerland over the last 35 years. J Hepatol 49:72-77

8. Bresson-Hadni S, Vuitton DA, Bartholomot B et al (2000) A twenty-year history of alveolar echinococcosis: analysis of a series of 117 patients from eastern France. Eur J Gastroenterol Hepatol 12:327-336

9. Kasai Y, Koshino I, Kawanishi N et al (1980) Alveolar echinococcosis of the liver; studies on 60 operated cases. Ann Surg 191:145-152

10. Dulger AC, Esen R, Begenik $H$ et al (2012) Alveolar echinococcosis of the liver: a single center experience. Pol Arch Med Wewn 122:133-138
11. Wilson JF, Rausch RL, Wilson FR (1995) Alveolar hydatid disease. Review of the surgical experience in 42 cases of active disease among Alaskan Eskimos. Ann Surg 221:315-323

12. Kadry Z, Renner EC, Bachmann LM et al (2005) Evaluation of treatment and long-term follow-up in patients with hepatic alveolar echinococcosis. Br J Surg 92:1110-1116

13. Buttenschoen K, Carli Buttenschoen D, Gruener B et al (2009) Long-term experience on surgical treatment of alveolar echinococcosis. Langenbecks Arch Surg 394:689-698

14. Kodama Y, Fujita N, Shimizu $T$ et al (2003) Alveolar echinococcosis: MR findings in the liver. Radiology 228:172-177

15. Becce F, Pomoni A, Uldry E et al (2014) Alveolar echinococcosis of the liver: diffusion-weighted MRI findings and potential role in lesion characterisation. Eur J Radiol 83:625-631

16. Dindo D, Demartines N, Clavien P-A (2004) Classification of surgical complications: a new proposal with evaluation in a cohort of 6336 patients and results of a survey. Ann Surg 240:205-213

17. Emre A, Ozden I, Bilge O et al (2003) Alveolar echinococcosis in Turkey. Experience from an endemic region. Dig Surg 20:301-305

18. McManus DP, Zhang W, Li J et al (2003) Echinococcosis. Lancet 362:1295-1304

19. Sato N, Namieno T, Furuya K et al (1997) Contribution of mass screening system to resectability of hepatic lesions involving Echinococcus multilocularis. J Gastroenterol 32:351-354

20. Liu YH, Wang XG, Gao JS et al (2009) Continuous albendazole therapy in alveolar echinococcosis: long-term follow-up observation of 20 cases. Trans R Soc Trop Med Hyg 103:768-778

21. Ammann RW, Fleiner-Hoffmann A, Grimm F et al (1998) Longterm mebendazole therapy may be parasitocidal in alveolar echinococcosis. J Hepatol 29:994-998

22. Ozturk G, Polat KY, Yildirgan MI et al (2009) Endoscopic retrograde cholangiopancreatography in hepatic alveolar echinococcosis. J Gastroenterol Hepatol 24:1365-1369

23. Sezgin O, Altintaş E, Saritaş U et al (2005) Hepatic alveolar echinococcosis: clinical and radiologic features and endoscopic management. J Clin Gastroenterol 39:160-167

24. Vogel J, Görich J, Kramme E et al (1996) Alveolar echinococcosis of the liver: percutaneous stent therapy in Budd-Chiari syndrome. Gut 39:762-764

25. Brunetti E, Kern P, Vuitton DA et al (2010) Expert consensus for the diagnosis and treament of cystic and alveolar echinococcosis in humans. Acta Trop 114:1-16

26. Ishizu H, Uchino J, Sato N et al (1997) Effect of albendazole on recurrent and residual alveolar echinococcosis of the liver after surgery. Hepatology 25:528-531

27. Ito A, Nakao M, Kutsumi H et al (1993) Serodiagnosis of alveolar hydatid disease by western blotting. Trans R Soc Trop Med Hyg 87:170-172

28. Liance M, Janin V, Bresson-Hadni S et al (2000) Immunodiagnosis of Echinococcus infections: confirmatory testing and species differentiation by a new commercial Western Blot. J Clin Microbiol 38:3718-3721

29. Gottstein B, Jacquier P, Bresson-Hadni S et al (1993) Improved primary immunodiagnosis of alveolar echinococcosis in humans by an enzyme-linked immunosorbent assay using the Em2plus antigen. J Clin Microbiol 31:373-376

30. Caoduro C, Porot C, Vuitton DA et al (2013) The role of delayed 18 F-FDG PET imaging in the follow-up of patients with alveolar echinococcosis. J Nucl Med 54:358-363 